\title{
Impact of a nutrition education programme on preschool children's willingness to consume fruits and vegetables
}

\author{
Emily M Melnick* @ , Karen Thomas, Charlotte Farewell, Jennie Quinlan, \\ Deanna LaFlamme, Diane Brogden, Sharon Scarbro and Jini E Puma \\ Rocky Mountain Prevention Research Center, Colorado School of Public Health, University of Colorado Anschutz \\ Medical Campus, Aurora, CO 80045, USA
}

Submitted 23 May 2019: Final revision received 1 November 2019: Accepted 3 December 2019: First published online 28 April 2020

\begin{abstract}
Objective: To evaluate the impact of a preschool-based nutrition education programme consisting of twelve 'hands on' nutrition education lessons delivered during the school year on young children's willingness to consume fruits and vegetables. Design: Quasi-experimental, pre-post design including the collection of plate waste evaluation data at the start and end of the 2015-2016 school year within two groups: (1) randomly selected classrooms receiving the intervention and (2) within conveniently sampled preschool classrooms not receiving the intervention serving as a comparison group.

Setting: Centre-based preschool programmes serving low-income families in the Denver metro area.

Participants: Three- to five-year-old children in preschool classrooms participating in the intervention during the 2015-2016 school year (n 308) and children enrolled in comparison classrooms ( $n$ 215).

Results: Repeated-measures logit models assessed whether increases in the odds of consuming small samples of fruits and vegetables between Time 1 (pre-intervention) and Time 2 (post-intervention) were different for children within the intervention group compared with the comparison group. Analyses showed that the change over time in consumption of the three vegetable samples varied by intervention status with greater change occurring among children within the intervention group (edamame: $P=0.001$; cauliflower: $P \leq 0.0001$ and red pepper: $P \leq 0.0001)$. Unlike vegetables, the change over time in consumption of the two fruit samples was not different between children within the intervention and comparison groups. Conclusions: An experiential-learning nutrition education programme can positively influence eating behaviours of low-income preschoolers in a centre-based setting by increasing willingness to consume vegetables.
\end{abstract}

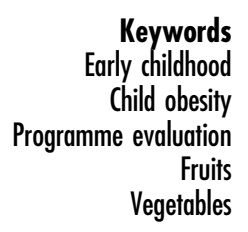

Few children aged 3-5 years meet the recommended dietary guidelines for fruit and vegetable consumption in the USA ${ }^{(1)}$. From an early age, children's experiences with fruits and vegetables influence both preferences and intake ${ }^{(2,3)}$. Evidence from laboratory studies suggests that when preschool-aged children are given repeated opportunities to taste unfamiliar foods, there is an increase in their affinity for, and consumption of, those foods ${ }^{(4-8)}$. Evidence also shows that limited exposure to a variety of foods may decrease acceptance of new foods. Increases in willingness to consume healthful foods, including fruits and vegetables, early in life impact later dietary preferences into adolescence and adulthood ${ }^{(9)}$. Therefore, the earlier and more varied the exposure, the healthier the child's diet will be throughout their life ${ }^{(10,11)}$. Establishing these healthy dietary preferences early in life is important because consuming recommended amounts of fruits and vegetables is associated with decreased risk for obesity ${ }^{(12)}$. Consequently, interventions which promote fruit and vegetable consumption during the early childhood years (aged 3-8 years) may have long-term benefits that reduce the likelihood of not only obesity but also other chronic diseases associated with obesity ${ }^{(8,9,13-15)}$.

The centre-based preschool care setting is an ideal location to introduce children to health-promoting foods including fruits and vegetables for a number of reasons. 
Over $50 \%$ of preschool-aged children spend time in centrebased care, so there is the potential to impact dietary behaviours of a large number of children through this channel of intervention ${ }^{(16)}$. Additionally, children attending full-time centre-based care consume up to two-thirds of their daily energetic intake in this environment, so it is an optimal time to introduce new healthful foods ${ }^{(16)}$. This may be particularly impactful for children living in poverty whose families are more likely to experience financial restrictions related to purchasing fruits and vegetables and who are more likely to live in communities with limited access to fruits and vegetables ${ }^{(17,18)}$.

Consequently, effective nutrition education programmes in preschool settings are needed to increase preschool children's willingness to try fruits and vegetables, especially among low-income and ethnic minority preschool-aged children who are disproportionately impacted by obesity ${ }^{(1)}$. Previously published results from nutrition education programmes delivered in preschool classrooms serving high-need families demonstrate success in changing children's dietary behaviours including willingness to taste novel fruits and vegetables ${ }^{(10,19-22)}$. In one study published in 2001, surveys completed by parents of preschoolers before and after an intervention indicated that child consumption of fruits and vegetables increased after a twelve-lesson theory-based nutrition education curriculum delivered in day care centres ${ }^{(20)}$. The Food Friends programme, a 12-week social marketing campaign aimed at encouraging preschool-aged children to try new foods, also demonstrated positive effects on child eating behaviours. Findings from a quasi-experimental design evaluating the Food Friends programme demonstrated increased preference for, and willingness to try, new foods among children in experimental preschool centres as measured by preference assessments, classroom observations and teacher surveys ${ }^{(19)}$. Finally, a randomised control trial conducted by Wardle et al. found that exposing 2-6 year olds to a less preferred vegetable over a 2 -week period resulted in increased affinity towards, and consumption of, that less preferred vegetable ${ }^{(10)}$.

Findings from these programmes suggest that simple exposure to novel foods in early childhood impacts children's preferences and consumption of fruits and vegetables and that this impact is significant regardless of geography, duration or curricula. These results are promising; however, further evidence using stringent evaluation techniques, which include objective pre-post measurements of child fruit and vegetable consumption and the inclusion of a comparison group, is needed to more fully evaluate the effectiveness of nutrition education programming in preschool settings.

This paper describes an evaluation study to test the impact of the Culture of Wellness in Preschools Nutrition Education (COWP-NE) programme component, delivered as twelve 30-45 min lessons over the course of the 20152016 school year, on preschool children's willingness to consume fruits and vegetables. Because this study includes objective pre-post measurements and a comparison group, findings extend previous research evaluating the effectiveness of nutrition education programming in preschool settings.

\section{Methods}

\section{Overview of Culture of Wellness in Preschools Nutrition Education}

The COWP programme is a comprehensive, early childhood obesity programme that comprises five programme components: (1) a classroom-based nutrition education (COWP-NE); (2) a classroom-based physical activity professional development and coaching (COWP-PA); (3) a staff workplace wellness programme (COWP-WW); (4) a parent wellness workshop series (COWP-PWW) and (5) a strategic planning process to make healthpromoting policy, system and environment changes (COWP-PSE). The COWP-NE programme component was adapted from the kindergarten version of the Integrated Nutrition Education Program for implementation in a centre-based preschool setting. Integrated Nutrition Education Program is a nutrition education programme that was developed by education and nutrition specialists, implemented and tested in an urban school setting, and proven to increase fruit and vegetable consumption among elementary-aged students ${ }^{(23,24)}$. The COWP programme team convened a group of high-performing preschool teachers to guide the adaptation process. The COWP team ensured that this group included members of cultural groups served by participating preschool centres to ensure lessons and lesson foods were culturally acceptable.

The resulting COWP-NE curriculum incorporated teacher recommendations and best practices related to nutrition education in preschool settings ${ }^{(25)}$. The curriculum additionally integrated theoretical frameworks including the Social Cognitive Theory and Piaget's Cognitive Development Theory ${ }^{(26,27)}$. COWP-NE lessons included: a nutrition question of the day, a developmentally appropriate book to be read to the preschool children, a song to be sung by the class related to healthy eating, an experiential food preparation and tasting activity and optional extension activities aligned with early learning frameworks. During the 'hands on' food preparation and tasting activities, children washed, prepared, served and tasted new foods including fruits, vegetables, low-fat dairy products and whole grains. Lessons also included a letter that was sent home to families with the recipe their child prepared and tried in the classroom that week.

Participating preschool classrooms received twelve COWP-NE lessons during the 2015-2016 school year. The names of these twelve lessons and food groups included in each are displayed in Table 1. Each COWP$\mathrm{NE}$ lesson lasted approximately 30-45 min depending on 
Table 1 Culture of Wellness nutrition education programme (COWP-NE) lesson titles and lesson foods served

\begin{tabular}{|c|c|}
\hline Lesson title & Lesson fruits and vegetables \\
\hline $\begin{array}{l}\text { 1. Germs are not for } \\
\text { sharing }\end{array}$ & Seasonal fruit \\
\hline 2. Manners & $\begin{array}{l}\text { Red bells pepper, lime, yellow } \\
\text { squash, zucchini squash, maize }\end{array}$ \\
\hline 3. Pumpkin circle & Pumpkin \\
\hline 4. Families & Cucumber, tomato \\
\hline 5. Healthy teeth & Carrot, snap pea, yellow pepper \\
\hline 6. Graphing favourite fruits & Orange, kiwi, pear, grape \\
\hline $\begin{array}{l}\text { 7. Graphing favourite } \\
\text { vegetables }\end{array}$ & $\begin{array}{l}\text { Radish, broccoli, snap pea, yellow } \\
\text { bell pepper }\end{array}$ \\
\hline 8. Today I ate a rainbow & $\begin{array}{l}\text { Spinach, red bell pepper, yellow } \\
\text { squash, carrot, grape, blueberry }\end{array}$ \\
\hline 9. Fruit pizza & Orange, blueberry, pineapple \\
\hline 10. Tortilla factory & $\begin{array}{l}\text { Green bell pepper, lime, tomato, } \\
\text { mango, maize }\end{array}$ \\
\hline 11. Fruity milkshake & Banana, strawberry \\
\hline 12. Vegetable bug & $\begin{array}{l}\text { Cucumber, carrot, spinach, } \\
\text { tomato, olive }\end{array}$ \\
\hline
\end{tabular}

whether or not teachers included optional extension activities. In aggregate, the total intervention dose for participating classrooms was approximately $6 \mathrm{~h}$ over the course of the school year (September 2015-May 2016).

Preschool teachers delivered COWP-NE lessons in the classroom after receiving training by the COWP programme team prior to start of the 2015-2016 school year. These trainings prepared teachers to deliver the curriculum and lasted approximately $2 \mathrm{~h}$. Teachers also received curriculum binders for their classrooms with step-by-step guides to delivering lessons. All lessons are publicly available on the COWP programme website (cowpprogram.com).

Because children living in low-income families are disproportionately affected for obesity, all preschool programmes selected to the COWP-NE programme served low-income families ${ }^{(28)}$. These programmes included Head Start programmes, a federally funded preschool programme in the USA which provides free care to low-income families with children aged 3-5 years old, as well as schoolbased and privately owned centre-based preschool programmes.

\section{Study design}

To evaluate the effect of COWP-NE on child willingness to consume selected samples of fruits and vegetables, evaluators from the COWP programme team conducted a quasiexperimental, pre-post design. The design included the collection of plate waste evaluation data at the start and end of the 2015-2016 school year within two groups: (1) randomly selected classrooms receiving the intervention and (2) within conveniently sampled preschool classrooms not receiving the intervention serving as a comparison group. The University of Colorado Institutional Review Board approved all study procedures with an expedited review. Implied consent was obtained from all participating classrooms. Evaluators distributed Memorandums of
Understanding outlining the evaluation process to all participating preschools which were then signed and returned by preschool directors. Evaluators noted first name and last initial of participating students during data collection to enable Time 1 and Time 2 matching, and data were then de-identified at the analysis stage.

\section{Participants and recruitment}

The study design included a stratified random sampling process to select preschool classrooms participating in COWP-NE in the Denver Metro area for inclusion in the intervention study group. Evaluators stratified preschool classrooms according to length of day (full-day $v$. half day) and type of centre (Head Start $v$. non-Head Start). This stratified random sampling strategy ensured that different types of centres and classrooms were represented proportionately within our sample. Using this technique, evaluators sampled thirty-three classrooms from a total of 171 classrooms who received the COWP-NE intervention in the 2015-2016 school year. Within this sample, eight classrooms were half-day non-Head Start classrooms, nine were half-day Head Start classrooms, eight were full-day non-Head Start classrooms and eight were full-day Head Start classrooms. This resulted in an initial sample size of 582 children who participated in evaluation activities for the intervention group.

Evaluators then recruited a conveniently sampled comparison group of preschool classrooms for inclusion in the study. This convenience sample of comparison group preschool classrooms participated in a separately funded physical activity programme (COWP-PA), but did not participate in the COWP-NE intervention component. Evaluators attempted to include all classrooms within twenty-two preschool centres who participated in this separately funded physical activity programme within our comparison group. Classrooms within ten preschool centres were excluded due to a combination of inability to schedule (centre, $n$ 4), being outside of the Denver Metro area (centre, $n 2$ ) and participation in separate nutrition education programmes during the school year (centre, $n 4$ ). This resulted in a comparison group sample of twelve preschool centres. All classrooms from these twelve preschools participated in the evaluation activities (classroom, $n$ 18). This resulted in an initial sample size of 341 children who did not receive the COWP-NE intervention participating in the evaluation activities as the comparison group of the study.

The final analytic sample excluded students who did not participate in both the Time 1 and Time 2 plate waste activities. Classrooms from three intervention centres and one comparison centre were additionally excluded from analyses due to mid-year centre closures. After exclusion of classrooms within these closed centres and exclusion of children missing either Time 1 or Time 2 data, the final analytic sample included 308 children in the intervention 
group and 215 children in the comparison group. Sample sizes for some specific food types varied slightly (up to eight children) due to either child allergies to sample foods or sample foods being dropped on the floor by children.

\section{Procedures}

To evaluate the impacts of the COWP-NE programme component on children's willingness to consume samples of specific fruits and vegetables, evaluators used a direct weighing plate waste method. In this method, each individual sample of food for each individual child is weighed before tasting and then weighed again after tasting to determine child consumption ${ }^{(29)}$.

Evaluators provided training on protocols to all COWP programme team members who conducted plate waste to ensure data collection was consistent across all preschools and classrooms. This training lasted $1 \mathrm{~h}$ and took place prior to any data collection activities. The protocol included a script with instructions for the children and staff. Additionally, evaluators provided pictures of the food samples (arrangement and size of samples in the serving tray) and measurements (in inches) to COWP programme team members conducting plate waste in order to maintain consistency in sample sizes. At each school, two trained COWP programme team members executed the plate waste evaluation activity. The same programme team member was responsible for weighing the samples before and after the tasting to maintain consistency.

Children who participated in the plate waste evaluation activity received a tray labelled with their first name and last initial for identification that was filled with five pre-weighed sample cups of food. Each tray included five cups filled with the following foods: (1) three edamame beans, (2) one slice of clementine, (3) one-half inch piece of cauliflower, (4) one-inch by half-inch strip of red pepper and (5) half of one strawberry. All non-edible portions of foods were removed prior to the plate waste evaluation activity. Evaluators selected red peppers because they were included in multiple COWP-NE lessons, cauliflower and edamame because they were assumed to be more novel foods, and oranges and strawberries because they were assumed to be more familiar foods to the children included in the evaluation groups. Edamame beans fall within the legume family, but are considered a part of the vegetable group by the US Department of Agriculture ${ }^{(30)}$. Consequently, edamame will be referred to as a vegetable within this paper. Programme team members conducting plate waste instructed children to taste anything they wanted to and to leave any foods they did not wish to taste in the sample cup. After children sampled the foods they wished to try, programme team members collected labelled trays and weighed the remaining food samples in a separate area.

Programme team members collected Time 1 (pre) plate waste data at intervention centres within 1 month prior to the start of the school-year intervention to determine baseline (Time 1: 9 September 2015-21 October 2015). Time 2 (post) data were then collected within 1 month after the conclusion of the school-year intervention (Time 2: 11 April 2016-24 May 2016). Time 1 plate waste data for the comparison group were collected between 9 February 2016 and 29 February 2016 and Time 2 data between 4 May 2016 and 27 May 2016. To further ensure standardisation, programme team members always collected plate waste data mid-morning (between breakfast and lunch) consistently across centres to avoid variation in the children's appetites. Additionally, data collection occurred at the same time of day at both Time 1 and Time 2 at the classroom level.

Programme team members recorded weights at the individual level for each sample. Percentage consumption was then determined by the weight difference in the original sample and remaining sample divided by the original sample weight. All samples were weighed on a digital scale accurate to the nearest gram (US BALANCE ${ }^{\circledR}$ model US-GLACIER, $250 \times 0.01 \mathrm{~g}$ ). To avoid measurement errors, programme team members weighed the original samples before tasting and the remaining samples after tasting in the same cup. After collection, programme research team members entered all data into Excel spreadsheets. Evaluators conducted a one-in-five verification procedure at the data entry level in which one in five classrooms was double-checked for data entry errors to ensure accuracy.

\section{Measures}

The outcome measures of interest were consumption percentages of each of the five sample fruits and vegetables. The change in these outcome measures from Time 1 to Time 2 indicated change in children's consumption of fruits and vegetables. These changes in consumption between Time 1 and Time 2 were compared between the intervention and comparison groups to determine the effect of the COWP-NE intervention on children's willingness to consume fruits and vegetables. Models assessed differences in percentage consumed rather than actual consumption amounts because the exact weight of any of the five fruit/vegetable samples may have differed slightly between pre-assessment and post-assessment.

\section{Data analysis}

To test the effect of the intervention on food consumption evaluators used a repeated-measures generalised linear regression model with a binomial distribution and a logit link function. The outcome of interest was consuming $\geq 90 \%$ of the food sample. Because a 'u-shaped' distribution for all food samples was observed (due to children either eating none/very little of the sample or most/all of the sample), evaluators decided to dichotomise the outcome for each food sample. Evaluators chose $90 \%$ 
consumption as a statistical cut-off to allow for measurement error and small amounts of food to be left in the cup.

Interaction terms incorporated in the models included group (intervention, comparison), time $(1,2)$ and time by group interaction, with the time by group interaction term indicating a difference by group in odds of Time 2 $v$. Time 1 . All models, except that for strawberry, included a random centre effect to account for the correlation within centres (interclass correlation coefficients $>0 \cdot 12$ ). The random effect for strawberry was estimated to be zero because most children ate $90 \%$ or more of the strawberry at all time points. Consequently, the random effect was removed from the model. All models also included fixed effects for percentage of students who qualified for free- or reduced-price lunches, percentage of white students and percentage of black students in order to control for the effects of these variables on change over time. Models used residual pseudo-likelihood estimation, an unstructured covariance matrix to model the random intercept effect for centre (G-side matrix) and a variance components matrix for the residuals (R-side matrix). An $\alpha$ was set at 0.05. The SAS proc glimmix procedure (SAS statistical software version 9.4 M6 (SAS Institute)) was used to run the regression models.

\section{Results}

\section{Study participants}

Table 2 presents family income and race/ethnicity data for the intervention and comparison groups. The table shows that classrooms within both the intervention and comparison preschool centres serve a majority of children who live in poverty as indicated by the percentage of children at the preschool centre who qualified for free- or reduced-price lunches ${ }^{(31)}$. If free- or reduced-price lunch data were unavailable for the preschool centre, evaluators used data from the nearest elementary school serving preschool-aged children. In the state of Colorado, children qualify for free lunches if their family's income is $<130 \%$ of the federal poverty level and for reduced price lunches if their family's

Table 2 Study participant demographics by study group (total $n 510$ )

\begin{tabular}{lcc}
\hline $\begin{array}{l}\text { Participant } \\
\text { demographics }\end{array}$ & $\begin{array}{c}\text { Intervention } \\
(n \text { 308) }\end{array}$ & $\begin{array}{c}\text { Comparison } \\
(n \text { 215 })\end{array}$ \\
\hline Centre type, (centre, $n)$ & & \\
Head start & 13 & 0 \\
Privately owned & 4 & 10 \\
School-based & 3 & 1 \\
Free and reduced & 96.0 & 71.1 \\
$\quad$ lunch (\%) & & \\
Race/ethnicity (\%) & 22.9 & 36.6 \\
Non-Hispanic white & 54.1 & 54.3 \\
Hispanic/Latino & 14.4 & 3.7 \\
Non-Hispanic black & 8.6 & 5.4 \\
Other & & \\
\hline
\end{tabular}

income is $<185 \%$ of the federal poverty level ${ }^{(31)}$. Because the percentage of children who qualified for free- or reduced-price lunches in the intervention group was greater than the percentage who qualified in the comparison group ( $95 \%$ in intervention group compared with $71.1 \%$ in comparison group), this variable was included as a fixed effect in the analytical model.

The percentage of Hispanic/Latino children was roughly the same between the comparison and intervention groups $(54.1 \%$ in intervention group compared with $54.3 \%$ in comparison group). The intervention group included a lower percentage of non-Hispanic white children $(22.9 \%$ in the intervention group compared with $36.6 \%$ in the comparison group) and a higher percentage of non-Hispanic black children (14.4\% in the intervention group compared with $3.7 \%$ in the comparison group). To control for this difference, the percentage of white students and the percentage of black students were also included as fixed effects in the analytical model.

\section{Outcomes}

To give context, the average weight in $g$ of foods prior to tasting averaged across both study groups was as follows: strawberries, 19.72 (SD 4.90) g; oranges 8.84 (SD 2.41) g; edamame, 4.40 (SD 0.56) g; cauliflower, 4.46 (SD 1.25) g and red peppers, 4.78 (SD 1.15) g. When results were analysed over time and by intervention/comparison groups, the results showed that the change over time in the odds of consumption of the three vegetable samples varied significantly by group status with greater change occurring among children within the intervention group, controlling for random (classroom) and fixed effects (\% of free- or reduced-price lunches, $\%$ of white students and $\%$ of black students) (edamame: $\mathrm{F}_{1,999}=9 \cdot 0, P=0 \cdot 0003$; cauliflower: $\mathrm{F}_{1,1003}=15.9, P \leq 0.0001$ and red peppers: $\mathrm{F}_{1,991}=26.4$, $P \leq 0.0001)$. This statistically significant difference in change over time by intervention status was not detected for the two fruit samples of strawberries and oranges. These data are displayed in Table 3.

\section{Discussion}

The present work evaluated the impact of the COWP-NE programme on willingness to consume fruits and vegetables among low-income preschool-aged children in a centre-based setting. Findings demonstrate that 1 year of the COWP-NE programme, which exposed preschool children to fruits and vegetables in 'hands on' nutrition education lessons, increased children's willingness to consume vegetables. Children who participated in the programme were more willing to taste and consume three types of vegetables compared with children who did not participate in the programme. This is evidenced by significantly greater changes over time in child consumption of vegetables over 


\section{Public Health Nutrition}

Table 3 Change over time in child consumption of sample fruits and vegetables by study group (total $n$ 523)

\begin{tabular}{|c|c|c|c|c|c|c|c|c|c|c|c|c|c|c|c|}
\hline \multirow[b]{2}{*}{$\begin{array}{l}\text { Food sample } \\
\text { type }\end{array}$} & \multicolumn{7}{|c|}{ Intervention } & \multicolumn{7}{|c|}{ Comparison } & \multirow[b]{2}{*}{$\begin{array}{l}\text { Difference in change over } \\
\text { time between intervention } \\
\text { and comparison } \dagger \ddagger \text {, test } F \\
\text { statistic and df, } P \text { value }\end{array}$} \\
\hline & $n$ & $\begin{array}{c}\text { Percentage } \\
\text { of children } \\
\text { who } \\
\text { consumed } \\
90 \% \text { or } \\
\text { more at } \\
\text { Time 1 }\end{array}$ & $95 \%$ C & $\begin{array}{l}\text { Percentage } \\
\text { of children } \\
\text { who } \\
\text { consumed } \\
90 \% \text { or } \\
\text { more at } \\
\text { Time 2 }\end{array}$ & $95 \%$ C & $\begin{array}{c}\quad \text { OR } \\
(\text { ref }=\text { Time 1) } \dagger\end{array}$ & $95 \%$ C & $n$ & $\begin{array}{c}\text { Percentage } \\
\text { of children } \\
\text { who } \\
\text { consumed } \\
90 \% \text { or } \\
\text { more at } \\
\text { Time } 1\end{array}$ & $95 \%$ C & $\begin{array}{l}\text { Percentage } \\
\text { of children } \\
\text { who } \\
\text { consumed } \\
90 \% \text { or } \\
\text { more at } \\
\text { Time 2 }\end{array}$ & $95 \%$ C & $\begin{array}{c}\text { OR } \\
(\text { ref }=\text { Time 1) } \dagger\end{array}$ & $95 \%$ C & \\
\hline Strawberry & 308 & $76 \cdot 9$ & $71 \cdot 8,81 \cdot 5$ & 82.5 & $77 \cdot 8,86 \cdot 6$ & 1.4 & $0.9,2 \cdot 1$ & 215 & $82 \cdot 3$ & $76 \cdot 6,87 \cdot 2$ & 84.7 & $79 \cdot 1,89 \cdot 2$ & 1.2 & $0.7,2 \cdot 0$ & $P=0.60 F_{1521}=0.3$ \\
\hline Orange & 306 & $25 \cdot 8$ & $21 \cdot 0,31 \cdot 1$ & 30.4 & $25 \cdot 3,35 \cdot 9$ & 1.3 & $0.9,1.8$ & 210 & 83.8 & $78.1,88.5$ & $84 \cdot 3$ & $78 \cdot 7,88.9$ & 1.0 & $0.6,1.8$ & $P=0.132 F_{1997}=2.3$ \\
\hline Edamame & 306 & $11 \cdot 1$ & $7 \cdot 8,15 \cdot 2$ & $27 \cdot 5$ & $22 \cdot 5,32 \cdot 8$ & 3.0 & $2 \cdot 0,4 \cdot 7$ & 211 & $46 \cdot 9$ & $40 \cdot 0,53.9$ & $56 \cdot 4$ & $49 \cdot 4,63 \cdot 2$ & 1.5 & $1 \cdot 0,2 \cdot 2$ & $P=.003^{*} F_{1,999}=9.0$ \\
\hline Cauliflower & 305 & 9.5 & $6 \cdot 5,13 \cdot 4$ & 29.8 & $24 \cdot 8,35 \cdot 3$ & 4.0 & $2 \cdot 6,6 \cdot 4$ & 214 & $45 \cdot 3$ & $38 \cdot 5,52 \cdot 3$ & 53.7 & $46 \cdot 8,60 \cdot 6$ & 1.4 & $1 \cdot 0,2 \cdot 1$ & $P \leq 0.001 * F_{1,1003}=15.9$ \\
\hline Red pepper & 300 & 8.7 & $5 \cdot 7,12 \cdot 4$ & 33.3 & $28 \cdot 0,39 \cdot 0$ & $5 \cdot 3$ & $3 \cdot 3,8 \cdot 4$ & 213 & $44 \cdot 6$ & $37 \cdot 8,51 \cdot 6$ & $54 \cdot 0$ & $47 \cdot 1,60 \cdot 8$ & 1.5 & $1 \cdot 0,2 \cdot 1$ & $\left.P \leq 0.001 * F_{1,991}=26.4\right)$ \\
\hline
\end{tabular}

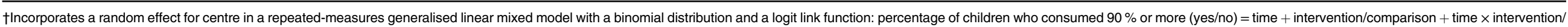
comparison + centre (random effect).

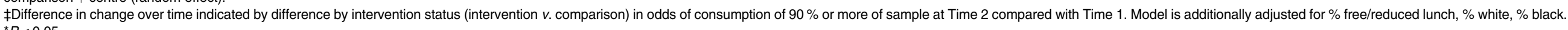
${ }^{*} P<0.05$ 
the school year among children within the intervention group compared with those within the comparison group.

While results indicate greater increases in consumption in the three samples of vegetables in the intervention group compared with the comparison group, analyses did not detect significant increases in the two fruit samples. Evaluators from the COWP team hypothesise that the lack of significant change in consumption for strawberries and oranges may have been due to a ceiling effect. Children consumed approximately $80 \%$ of oranges and strawberries served at baseline, leaving little room for improvement. This high level of consumption at Time 1 may have been due to the fact that children were more familiar with oranges and strawberries at the beginning of the school year and thus more willing to taste these foods. This is not surprising given previously published evidence that preschool-aged children prefer fruits compared with vegetables ${ }^{(1,32)}$. Consequently, educators may want to select foods that are less familiar at baseline in future evaluation studies.

These results are consistent with previous studies of nutrition education programmes in preschool settings which demonstrate the influence of exposure to vegetables ${ }^{(33-36)}$. However, many of these programme evaluations relied on subjective assessments of dietary behaviour change. The present study fills this gap by using an objective goldstandard assessment of dietary behaviour change.

\section{Strengths and limitations}

Strengths of this study include the use of an objective plate waste collection method to determine increased willingness to consume fruits and vegetables. Utilisation of a quasi-experimental, pre-post design minimised possible confounding covariates.

A limitation that should be noted is the difference in the time of Time 1 plate waste data collection between intervention and comparison groups. While programme team members collected intervention group Time 1 plate waste data in the September and October, comparison group Time 1 data were collected the following February. It is possible that this time difference may have implications for findings. Additionally, the percentage of children who qualified for free- or reduced-price lunches and the percentage of children who were black were lower in preschool centres within the comparison group compared with those in the intervention group. Because of these differences, evaluators included the percentage of students who qualified for free- or reduced-price lunches, the percentage of white students and the percentage of black students as fixed effects in the analytical model, but these differences might also have implications on the study's findings. Lastly, because this was a quasi-experimental design rather than a randomised trial, generalisability of the findings is limited. Nonetheless, the inclusion of a comparison group of preschool classrooms lends more confidence to the attribution of the increased willingness to consume vegetables among classrooms that received COWP-NE to the intervention itself.

\section{Conclusions}

The current study extends previous research findings suggesting that exposure to novel foods in preschool classroom settings through interactive nutrition education programmes can be associated with children's willingness to consume vegetables.

Increases in willingness to consume vegetables among children who participated in the COWP-NE programme hold particular significance because this sample consists of primarily low-income and ethnic minority children who are disproportionately affected by childhood obesity and its related co-morbidities. As such, the public health and nutrition education community should consider further interventions to provide young children, particularly those children disproportionately affected by obesity, the opportunities to taste foods in a preschool classroom setting. Given the inadequate consumption of vegetables among young children and childhood obesity prevalence at epidemic rates in the USA, implementing interventions to decrease risk for obesity and impact obesity-related behaviours based upon empirical evidence is essential to improve public health.

\section{Acknowledgements}

Acknowledgements: The authors extend a sincere thank you to all the participating preschool centres, children, teachers and administration, without whom this project would not have been possible. We additionally would like to acknowledge the research team members within the Rocky Mountain Prevention Research Center who helped to collect and enter data. Financial support: This work was supported by The Colorado Health Foundation (9115, PI: Dr. Jini Puma). The Colorado Health Foundation had no role in the design, analysis or writing of this article. Conflict of interest: None. Authorship: J.E.P., S.S., C.F. and D.L. designed the evaluation study. S.S. and J.E.P. analysed the data and interpreted results. E.M.M. primarily authored the manuscript. K.T., C.F., J.Q. and D.B. drafted sections of the manuscript and provided critical edits for the final version. Ethics of buman subject participation: This study was conducted according to the guidelines laid down in the Declaration of Helsinki, and all procedures involving research study participants were approved by the University of Colorado Institutional Review Board. (\#12-0902). Passive consent was obtained from all participants. The COWP research team distributed Memorandums of Understanding outlining the evaluation 
process to all participating preschools which were then signed and returned by preschool directors. Researchers noted first name and last initial of participating students during data collection to enable Time 1 and Time 2 matching and data were then de-identified at the analysis stage.

\section{References}

1. Kim SA, Moore LV, Galuska D et al. (2014) Vital signs: fruit and vegetable intake among children-united states, 2003-2010. MMWR Morb Mortal Wkly Rep 63, 671-676.

2. Pérez-Rodrigo C \& Aranceta J (2001) School-based nutrition education: lessons learned and new perspectives. Public Health Nutr 4, 131-139.

3. Schmitt SA, Bryant LM, Korucu I et al. (2019) The effects of a nutrition education curriculum on improving young children's fruit and vegetable preferences and nutrition and health knowledge. Public Health Nutr 22, 28-34.

4. Pliner P, Pelchat M \& Grabski M (1993) Reduction of neophobia in humans by exposure to novel foods. Appetite 20, 111-123.

5. Cooke L (2007) The importance of exposure for healthy eating in childhood: a review. J Hum Nutr Diet 20, 294-301.

6. Birch LL \& Marlin DW (1982) I don't like it; I never tried it: effects of exposure on two-year-old children's food preferences. Appetite 3, 353-360.

7. Birch LL, McPhee L, Shoba B et al. (1987) What kind of exposure reduces children's food neophobia?: looking vs. tasting. Appetite 9, 171-178.

8. Skinner JD, Carruth BR, Bounds W et al. (2002) Do foodrelated experiences in the first 2 years of life predict dietary variety in school-aged children? J Nutr Educ Behav 34, 310-315.

9. Russell CG, Taki S, Laws R et al. (2016) Effects of parent and child behaviours on overweight and obesity in infants and young children from disadvantaged backgrounds: systematic review with narrative synthesis. BMC Public Health 16, 151.

10. Wardle J, Cooke LJ, Gibson EL et al. (2003) Increasing children's acceptance of vegetables; a randomized trial of parentled exposure. Appetite 40, 155-162.

11. Birch LL (1979) Preschool children's food preferences and consumption patterns. J Nutr Educ 11, 189-192.

12. Rolls BJ, Ello-Martin JA \& Tohill BC (2004) What can intervention studies tell us about the relationship between fruit and vegetable consumption and weight management? Nutr Rev 62, 1-17.

13. Ben-Shlomo Y \& Kuh D (2002) A Life Course Approach to Chronic Disease Epidemiology: Conceptual Models, Empirical Challenges and Interdisciplinary Perspectives. Oxford: Oxford University Press.

14. Shanahan MJ (2000) Pathways to adulthood in changing societies: variability and mechanisms in life course perspective. Annu Rev Sociol 26, 667-692.

15. Ledoux T, Hingle M \& Baranowski T (2011) Relationship of fruit and vegetable intake with adiposity: a systematic review. Obes Rev 12, e143-e150.

16. Robson SM, Khoury JC, Kalkwarf HJ et al. (2015) Dietary intake of children attending full-time child care: what are they eating away from the child-care center? J Acad Nutr Diet 115, $1472-1478$.
17. Monsivais P, Aggarwal A \& Drewnowski A (2012) Are socioeconomic disparities in diet quality explained by diet cost?. $J$ Epidemiol Community Health 66, 530-535.

18. Drewnowski A (2004) Obesity and the food environment: dietary energy density and diet costs. Am J Prev Med 27, 154-162.

19. Johnson SL, Bellows L, Beckstrom L et al. (2006) Evaluation of a social marketing campaign targeting preschool children. Am J Health Behav 31, 44-55.

20. Cason KL (2001) Evaluation of a preschool nutrition education program based on the theory of multiple intelligences. J Nutr Educ 33, 161-164.

21. Sweitzer SJ, Briley ME, Roberts-Gray C et al. (2010) Lunch is in the bag: increasing fruits, vegetables, and whole grains in sack lunches of preschool-aged children. J Am Diet Assoc 110, 1058-1064.

22. Hassapidou M, Daskalou E, Paschaleri A et al. (2012) A nutrition intervention program in preschool children in northern Greece. Public Health Nutr 15, 1524-1525.

23. Auld GW, Romaniello C, Heimendinger J et al. (1998) Outcomes from a school-based nutrition education program using resource teachers and cross-disciplinary models. J Nutr Educ 30, 268-280.

24. Auld GW, Romaniello C, Heimendinger J et al. (1999) Outcomes from a school-based nutrition education program alternating special resource teachers and classroom teachers. J Sch Health 69, 403-408.

25. Baker S, Auld G, MacKinnon C et al. (2014) Best Practices in Nutrition Education for Low-Income Audiences. Washington, DC: National Institute of Food and Agriculture.

26. Bandura A \& Walters RH (1977) Social Learning Theory. Englewood Cliffs, NJ: Prentice-Hall.

27. Piaget J (1964) Part I: cognitive development in children: Piaget development and learning. J Res Sci Teach 2, 176-186.

28. Skinner AC, Ravanbakht SN, Skelton JA et al. (2018) Prevalence of obesity and severe obesity in US children, 1999-2016. Pediatrics 141, e20173459.

29. Liz Martins M, Cunha LM, Rodrigues SS et al. (2014) Determination of plate waste in primary school lunches by weighing and visual estimation methods: a validation study. Waste Manag 34, 1362-1368.

30. U.S. Department of Agriculture (2019) Beans Peas are Unique Foods. https://www.choosemyplate.gov/vegetablesbeans-and-peas (accessed June 2019).

31. Child Nutrition Programs - Income Eligibility Guidelines (2015) Fed Regist 80, 17026-17027.

32. Krølner R, Rasmussen M, Brug J et al. (2011) Determinants of fruit and vegetable consumption among children and adolescents: a review of the literature. Part II: qualitative studies. Int J Behav Nutr Phys Act 8, 112.

33. Gripshover SJ \& Markman EM (2013) Teaching young children a theory of nutrition: conceptual change and the potential for increased vegetable consumption. Psychol Sci 24, 1541-1553.

34. Holley CE, Farrow C \& Haycraft E (2017) A systematic review of methods for increasing vegetable consumption in early childhood. Curr Nutr Rep 6, 157-170.

35. Nekitsing C, Hetherington MM \& Blundell-Birtill P (2018) Developing healthy food preferences in preschool children through taste exposure, sensory learning, and nutrition education. Curr Obes Rep 7, 60-67.

36. Witt KE \& Dunn C (2012) Increasing fruit and vegetable consumption among preschoolers: evaluation of Color Me Healthy. J Nutr Educ Behav 44, 107-113. 